\title{
Continuous dynamics of color categorization
}

\author{
Stephanie Huette \\ University of California, Merced, California \\ AND \\ Bob McMurRaY \\ University of Iowa, Iowa City, Iowa
}

\begin{abstract}
In order to assess whether color categorization is sensitive to within-category differences in hue, we monitored mouse trajectories in a modified categorization task. Participants saw color swatches from a blue-green continuum and categorized them with a computer mouse by selecting one of two colored regions at the top of a monitor. An analysis of the mouse trajectories showed that the deviation toward the competing category was a function of hue: As hues approached the category boundary, they increasingly deviated to the competitor. This work presents evidence for parallel activation on the level of hue and category processing for color, as well as simultaneous activation of perceptually adjacent categories. Thus, a dynamic process sensitive to fine-grained within-category detail best characterizes color categorization.
\end{abstract}

Perceptual categorization is of fundamental interest in cognitive science, perhaps because of the mysterious tie between the physical world's lack of invariant cues and the qualitative distinctions observed in behavioral measures such as identification. Color categorization distills this to its core: a continuous dimension (hue) carved into multiple categories. Although many studies have examined the nature and structure of these categories (e.g., Pilling, Wiggett, Özgen, \& Davies, 2003; Rosch, 1975) and their possible interaction with the encoding of hue (e.g., Bornstein \& Korda, 1984), few have examined the process of arriving at a categorical response.

One factor useful in distinguishing models of categorization is the degree to which categorization preserves continuous within-category differences in parallel to categorical representations, or whether such distinctions are lost over time (McMurray, Tanenhaus, \& Aslin, 2009; Oden \& Massaro, 1978). In color, it is clear that people can perceive hue differences within a category. However, it is not known whether such differences affect categorization. The present study addressed this, asking whether fine-grained hue differences are preserved in parallel with categorical representations during forced choice categorization. This was assessed by examining moment-by-moment motor trajectories, as participants performed a discrete, forced choice identification task.

Early work on color categories (e.g., Rosch, 1975) showed that color terms prime visual percepts and have some form of prototype structure. More recently, there has been interest in the spontaneous influence of linguistic labels on color perception in purely perceptual tasks (e.g., discrimination), particularly in lateralization paradigms (Drivonikou et al.,
2007; Franklin et al., 2008; Gilbert, Regier, Kay, \& Ivry, 2006). These lines of work emphasize top-down interactions from language, not purely perceptual categories, and do not discuss the process of categorization.

The bulk of prior work on color categorization employs categorical perception (CP) (Bornstein \& Korda, 1984; Gilbert et al., 2006; but see Hampton, Estes, \& Simmons, 2005). CP is observed when discrimination is better across a category boundary than within a category, for equal physical distances (Liberman, Harris, Hoffman, \& Griffith, 1957; but see Schouten, Gerrits, \& van Hessen, 2003). Discrimination is crucial for establishing CP, but it emphasizes sensory or perceptual levels of processing and, in the CP framework, whether categories can influence this level. Thus, it can tell us little about categorization itself. This has led to important but unresolved debates about what levels (visual/linguistic) of categories are present and when they exert their influences on perception (Roberson \& Davidoff, 2000; Uchikawa \& Shinoda, 1996) but has not shed light on the nature of color categorization processes.

One explanation of CP is that categorization occurs independently and in parallel to continuous hue processing (Bornstein \& Korda, 1984; see also Pisoni \& Tash, 1974). Since categories are useful for processes such as language (Roberson \& Davidoff, 2000) and continuous hues form the basis of most visual processes, it makes sense to preserve both. This accounts for participants' abilities to both perceive within-category differences and show categorical effects on discrimination, since a cross-category comparison has two sources (hue and category) of difference to support it. This model is also supported by reaction times (RTs) in discrimination tasks (Bornstein \& Korda, 1984): 
Discrimination when there are both hue and category differences (between categories) will be faster than that with hue differences alone (within category) because two differences contribute to the former. It is also consistent with demonstrations that $\mathrm{CP}$ is affected by memory loads (Pilling et al., 2003; Roberson \& Davidoff, 2000) and is eliminated in tasks with no linguistic or memory demands (Roberson, Hanley, \& Pak, 2009).

According to this model, CP may only reflect the use of multiple sources of information for discrimination. It also suggests that within-category detail is available to contribute to categorization. However, there are no explicit tests of this parallelism. RT measures necessarily occur after processing. As a result, a serial model in which hue is processed prior to categorization (and perhaps subsequently discarded in favor of the category) could account for differences in RTs. Indeed, electrophysiological studies suggest that color categorization occurs with the same time course as other visual categories (Fonteneau \& Davidoff, 2007), rather than at earlier stages. This could support a serial process in which a category is not available until late. Our goal, then, is to determine whether both sources of information (hue and category) are available simultaneously in categorization.

One way to show continuous processing of distinct streams of information is through continuous motor trajectories. Spivey, Grosjean, and Knoblich (2005) first demonstrated this in word recognition. For example, when candy is heard, candle is briefly active in parallel (until $-d y$ is heard). As participants moved a mouse toward a picture of the target (candy), their trajectories veered momentarily toward the competitor, reflecting this parallel activation. This technique has been used to examine parallelism in sentence processing (Farmer, Anderson, \& Spivey, 2007) and categorization (Dale, Kehoe, \& Spivey, 2007; Freeman, Ambady, Rule, \& Johnson, 2008). Moreover, deviation toward the competitor is observed in early portions of the trajectories, even before the end of disambiguating words in sentence-processing studies. This indicates that intermediate stages of language processing (Farmer et al., 2007) and categorization (Dale et al., 2007; Freeman et al., 2008) are shared with motor systems.

There is also evidence of color processing being rapidly shared with motor processing (Schmidt, 2002). A control condition used in Farmer et al. (2007) showed mousetracking evidence that multiple color categories are active simultaneously. With a green stimulus, they observed curvature toward a blue box, but not toward a red one. Thus, adjacent color categories exhibit a hint of simultaneous, competitive activation. What is not known is whether this is sensitivity to competition between discrete categories nearby in color space or sensitivity to continuous, withincategory differences in hue.

In our task, participants categorized briefly presented color swatches varying along a green-blue continuum by selecting a prototypical green or blue colored box at the top of the screen. Sensitivity to hue differences should appear in the mouse trajectory as a linear increase in the amount of deviation toward the competitor object as the stimulus
Table 1

Target Munsell Hue and Resultant CIE $x, y, z$ Coordinates

\begin{tabular}{|c|c|c|}
\hline Step Number & Hue Value/Chroma & Resultant $x, y, z$ \\
\hline 1 & $10 \mathrm{~B} 6 / 6$ & $22.061,27.365,36.346$ \\
\hline 2 & $7.5 \mathrm{~B} 6 / 6$ & $21.048,27.554,35.699$ \\
\hline 3 & $5 \mathrm{~B} 6 / 6$ & $21.487,28.030,34.662$ \\
\hline 4 & $2.5 \mathrm{~B} 6 / 6$ & $19.107,27.524,32.559$ \\
\hline 5 & 10BG $6 / 6$ & $18.774,27.631,30.591$ \\
\hline 6 & $7.5 \mathrm{BG} 6 / 6$ & $18.686,27.881,28.317$ \\
\hline 7 & $5 \mathrm{BG} 6 / 6$ & $18.134,27.652,23.828$ \\
\hline 8 & $2.5 \mathrm{BG} 6 / 6$ & $18.848,28.024,23.874$ \\
\hline 9 & $10 \mathrm{G} 6 / 6$ & $18.918,28.369,20.801$ \\
\hline 10 & $7.5 \mathrm{G} 6 / 6$ & $19.559,28.702,40.403$ \\
\hline 11 & $5 \mathrm{G} 6 / 6$ & $19.739,28.818,19.125$ \\
\hline
\end{tabular}

approaches the category boundary. That is, participants should show more deviation to hues near the boundary than to prototypical values, and the amount of deviation should vary linearly with step. If such gradient responding were embedded in a categorical task, this would constitute strong evidence that both sources of information are available simultaneously, even in a task that would appear to restrict attention to categories.

\section{METHOD}

\section{Participants}

Fifty-eight people participated in this experiment and were compensated with course credit, in accordance with ethical guidelines at the University of California, Merced. The participants had normal color vision, had native English-speaking proficiency, and were right-handed or ambidextrous by self-report.

\section{Stimuli and Design}

The stimuli were adapted from Bornstein and Korda (1984) and consisted of an 11-step blue-green continuum. These were created in Munsell space and were calculated with a standard (1931) 2-degree observer. The continuum began at $10 \mathrm{~B}$ and ended with $5 \mathrm{G}$, with hue steps of 2.5 (see Table 1). Saturation and value were kept constant at 6 across the continuum. The experiment was conducted on a CRT monitor operating at $1,024 \times 768$ pixels, $60 \mathrm{~Hz}$ in a room with no other light source. The speed of the mouse was set to the second lowest bar in Mac OS X system settings, to reduce erratic movement.

There were 42 repetitions of each hue, for a total of 462 trials. The assignment of colors to response side was switched halfway through the experiment, and the order of these blocks was counterbalanced across participants (order is referred to as condition).

\section{Procedure}

On each trial (Figure 1), the endpoints of the continuum appeared at the top corners of a black screen, in $200 \times 200$ pixel squares. A $35 \times 35$ pixel red button appeared at the bottom of the screen $500 \mathrm{msec}$ after trial onset. The participants were instructed to click the button, at which time the endpoint pictures were reduced to $50 \times 50$ pixels. This increased the difficulty and minimized variance in the endpoint of the trajectory.

After clicking the button, the participants moved the mouse vertically toward the two targets. When the mouse moved over a hidden window (257 pixels from the bottom of the screen), the target stimulus was presented for $50 \mathrm{msec}$. The participants were instructed to continue the movement and select the responses most similar to the one they had seen flashed, by moving the mouse to their response. Clicking the final response was not necessary, preventing the participants from revising their decision. Mouse position was 


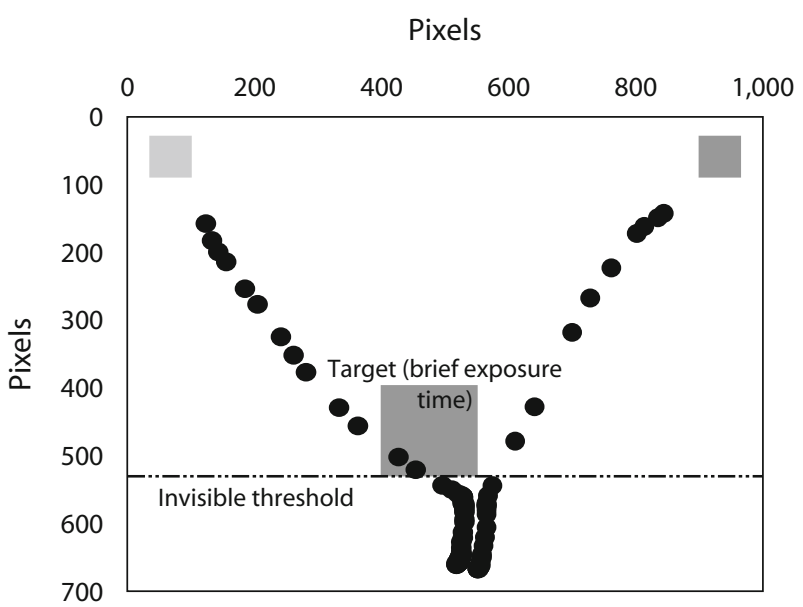

Figure 1. Experimental task. After the participants moved their mouse vertically over an invisible threshold, the target color was flashed briefly. The participants then began moving toward either green or blue to make their response (colors are presented in grayscale). The black dots represent samples from two separate trials.

sampled approximately every $15 \mathrm{msec}(67 \mathrm{~Hz})$, and the final selection (and the RT) was recorded as a measure of the participants' overt identification.

The 50-msec presentation was long enough to get an impression of the hue, without drawing sustained attention to the center of the screen. In piloting, longer durations increased the chance of stopping the mouse on the stimulus. The participants were instructed to smoothly move to one response or the other and were monitored by the experimenter for the first few trials to ensure that they understood the task and made smooth movements. The participants used their dominant hand to perform the task.

\section{Mouse-Tracking}

As in Spivey et al. (2005), $x, y$ mouse points were collected using the "mouse-move" event in PsyScope (Cohen, MacWhinney, Flatt,
\& Provost, 1993). In addition, response latency (from target onset to response), as well as final identification, was recorded.

To cope with variability in the RT, the time stamp on each sample was recoded as a percentage of the total RT and was resampled at increments of $5 \%$ by linear interpolation.

After time normalization, trials on which the response was on the left were mirror-reversed horizontally so that they could be averaged with those on the right. Next, to minimize variability in the starting position, the entire trajectory on each trial was translated so as to begin at the same Cartesian coordinates of $(0,0)$. Finally, the axes were rotated by a fixed angle, such that the endpoint was near 0 along the $y$-axis. This procedure transformed the $x$-axis into a straight line from the button to the response. Thus, the maximum $y$-coordinate becomes the maximum deviation from a straight path to the response.

\section{RESULTS}

\section{Identification}

The first analyses examined the ultimate identification response on each trial (which box was selected). The participants exhibited identification and RTs similar to those in Bornstein and Korda (1984) (see Figure 2). Logistic functions were fit to each participant's data and showed a boundary at step $5.9(S D=0.5)$. Eight participants were excluded for having boundaries greater than two standard deviations from the mean, because of experimenter error, or because they failed to correctly categorize the endpoints on more than $10 \%$ of the trials.

\section{Mouse Trajectories}

To assess the availability of within-category differences, we examined the deviation of the mouse trajectories from a straight line as a function of continuum step. Again, we should observe the lowest deviation for endpoint values and higher deviations as the hue approached the boundary. However, apparent differences within a category could be an artifact of averaging. Thus, we adapted two techniques

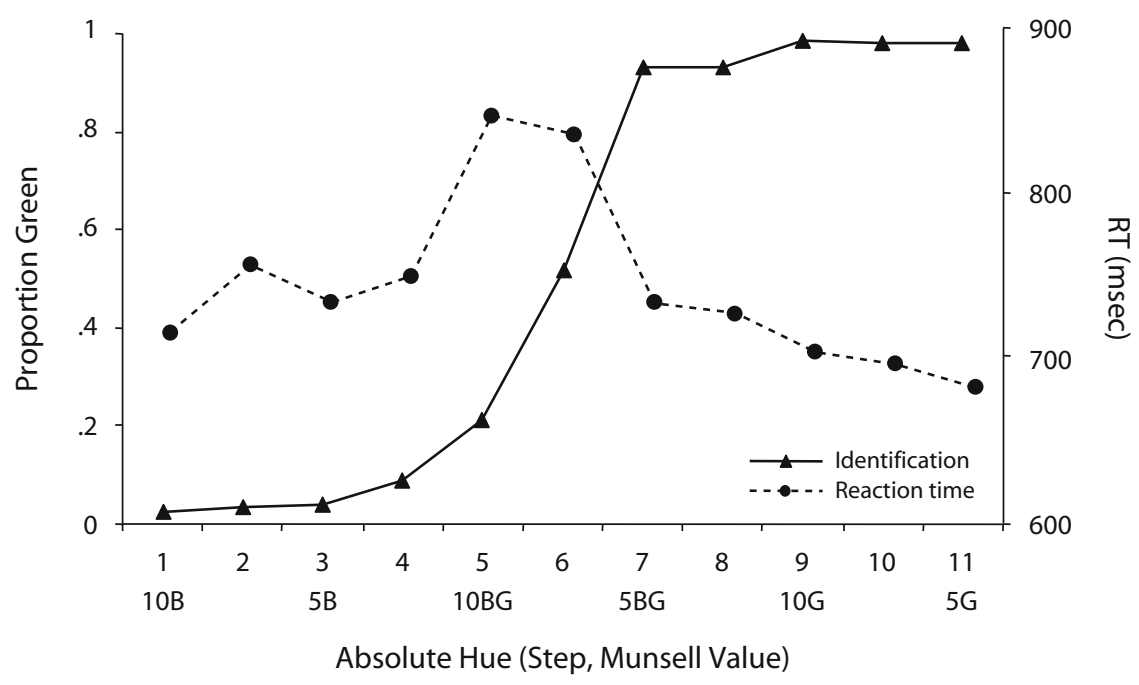

Figure 2. Proportions of trials on which green was selected, and reaction times (RTs) as a function of hue. Step 1 is the prototypical endpoint blue, and Step 11 is the endpoint green. Identification is plotted on the left $y$-axis, RT on the right. The abrupt transitions between categories and RT differences are consistent with the results from Bornstein and Korda (1984). 
Table 2

Representative Conversions From Step to rStep for 5 Participants

\begin{tabular}{|c|c|c|c|c|c|c|c|c|c|c|}
\hline & \multicolumn{10}{|c|}{ Participant No. } \\
\hline & \multicolumn{2}{|c|}{6} & \multicolumn{2}{|c|}{7} & \multicolumn{2}{|c|}{8} & \multicolumn{2}{|c|}{12} & \multicolumn{2}{|c|}{19} \\
\hline & Step & rStep & Step & rStep & Step & rStep & Step & rStep & Step & rStep \\
\hline Boundary & \multicolumn{2}{|c|}{5.41} & \multicolumn{2}{|c|}{6.08} & \multicolumn{2}{|c|}{5.74} & \multicolumn{2}{|c|}{7.1} & \multicolumn{2}{|c|}{6.4} \\
\hline \multirow[t]{7}{*}{ Blue } & & & & & & & 1 & -6.1 & & \\
\hline & & & 1 & -5.08 & & & 2 & -5.1 & 1 & -5.4 \\
\hline & 1 & -4.41 & 2 & -4.08 & 1 & -4.74 & 3 & -4.1 & 2 & -4.4 \\
\hline & 2 & -3.41 & 3 & -3.08 & 2 & -3.74 & 4 & -3.1 & 3 & -3.4 \\
\hline & 3 & -2.41 & 4 & -2.08 & 3 & -2.74 & 5 & -2.1 & 4 & -2.4 \\
\hline & 4 & -1.41 & 5 & -1.08 & 4 & -1.74 & 6 & -1.1 & 5 & -1.4 \\
\hline & 5 & -0.41 & 6 & -0.08 & 5 & -0.74 & 7 & -0.1 & 6 & -0.4 \\
\hline \multirow[t]{6}{*}{ Green } & 6 & +0.59 & 7 & +0.92 & 6 & +0.26 & 8 & +0.9 & 7 & +0.6 \\
\hline & 7 & +1.59 & 8 & +1.92 & 7 & +1.26 & 9 & +1.9 & 8 & +1.6 \\
\hline & 8 & +2.59 & 9 & +2.92 & 8 & +2.26 & 10 & +2.9 & 9 & +2.6 \\
\hline & 9 & +3.59 & 10 & +3.92 & 9 & +3.26 & 11 & +3.9 & 10 & +3.6 \\
\hline & 10 & +4.59 & 11 & +4.92 & 10 & +4.26 & & & 11 & +4.6 \\
\hline & 11 & +5.59 & & & 11 & +5.26 & & & & \\
\hline
\end{tabular}

from McMurray, Aslin, Tanenhaus, Spivey, and Subik (2008).

First, since boundaries vary between participants, gradiency in average trajectories could arise if the participants showed discrete categorization but boundaries varied. Thus, for each participant, continuum steps were recomputed as distance from the category boundary (relative step, or rStep) by subtracting each step from that participant's boundary (see Table 2). Steps closest to the boundary became steps +1 and -1 , with negative steps indicating the blue side and positive steps the green.

Second, even robustly green tokens (e.g., rSteps of 2-4) would be occasionally identified as blue (and vice versa). Such miscategorizations are more likely near the boundary and could contribute to gradiency in the averaged trajectories. Thus, for negative rSteps, we analyzed only trials on which the participants ultimately selected blue, and for positive rSteps, only green trials. This is analogous to including only trials on which a correct response was made (as defined by the participants' own boundary). For the green half of the continuum, we excluded $9 \%$ of the data, and for blue, $6 \%$ of the data. These techniques allow our test of gradiency to be conditioned on a set of maximally discrete categorizations.

Average trajectories (after rotation) can be seen in Figure 3 . The starting location of the mouse was at $(0,0)$, and the eventual choice was around $(1,000,50)$, making the $x$-axis an idealized path directly to the response. As rStep approached 0 (the boundary), there was increased pull toward the competitor (upward deviation) for both the blue and green sides of the continuum. This was quantified as the maximum deviation (maximum $y$ ) for each trial, which was then averaged within rStep for each participant.

In assessing this statistically, a standard ANOVA is problematic. First, continuous distance from the boundary (rStep) must be binned into discrete groups, potentially ignoring important variations. Second and more important, we cannot examine each participant's full range of stimuli. If one participant had six steps on the green side, but another had only four, we could analyze only the first four from both (otherwise, there would be missing cells). Thus,
A

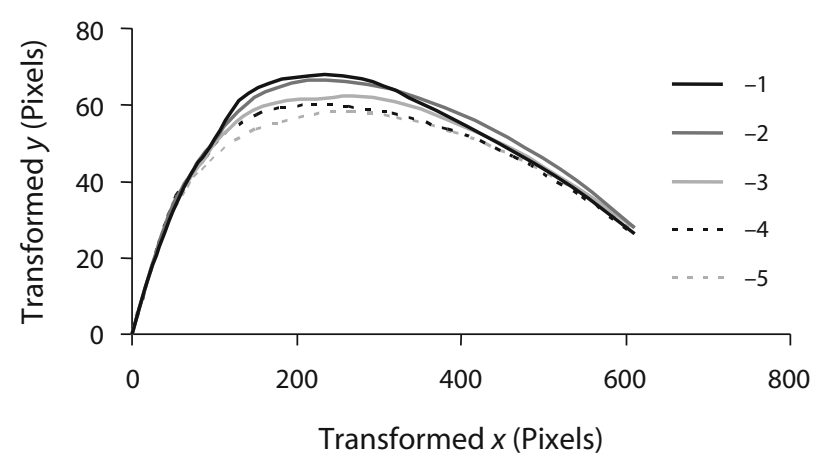

B

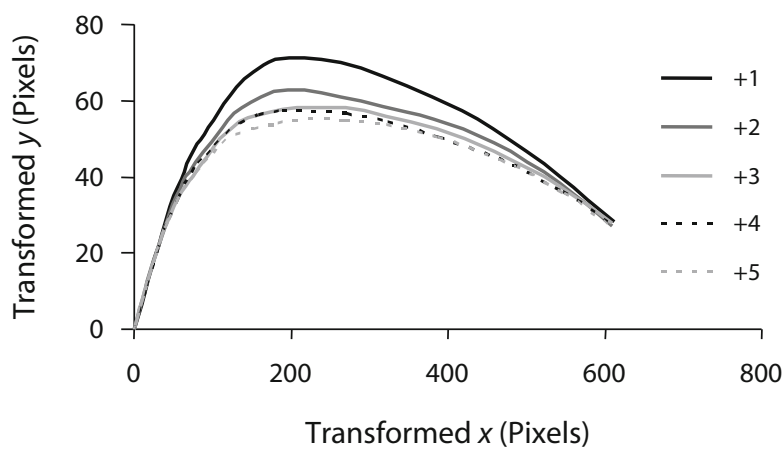

Figure 3. Transformed mouse trajectories as a function of distance from the category boundary. Note that the trajectories have been mirror reversed and rotated as described in the Method section, meaning that the $x$-axis represents a straight path to the response. (A) Responses to blue as a function of distance from the category boundary. Only tokens on the blue side of the continuum are shown (negative rSteps). (B) Responses to green. 


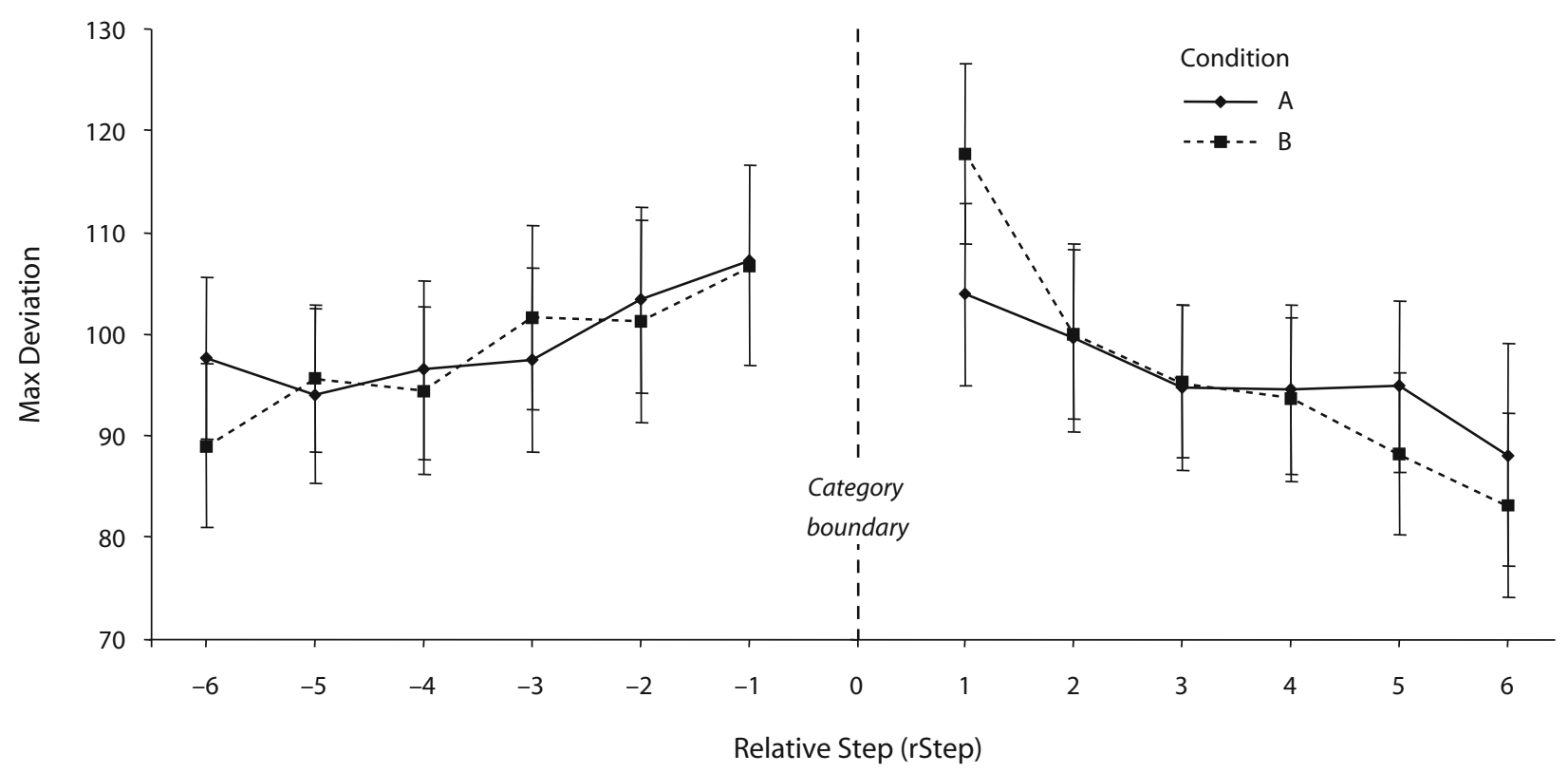

Figure 4. Averaged maximum deviation as a function of rStep and condition. Condition corresponds to whether green was presented on the left first (A) or second (B). As the rStep approaches the boundary, trajectories increasingly deviate from a straight line. Error bars represent $S E M$.

as in McMurray et al. (2008), hierarchical linear regressions were used (separately for blue and green rSteps), in which rStep was treated as a continuous covariate of the maximum deviation. Our primary concern, then, was the effect of rStep on deviation. This can be seen in Figure 4: As rStep approaches the category boundary from either side, the deviation increases.

Regressions were run hierarchically and included condition (whether the green response was on the left or the right first for that participant) as a between-subjects variable, 49 dummy codes to account for subject variance, rStep as a continuous within-subjects covariate, and an rStep $\times$ condition interaction. We adjusted both the $F$ statistic and the degrees of freedom on the within-subjects variables according to Cohen and Cohen's (1983) recommendations: $R^{2}$ and $F$ were computed with respect to participant variance only (not the total variance); the $F$ statistic was computed from this adjusted $R^{2}$ but had $n-1$ degrees of freedom (where $n$ is the number of participants, not data points).

The first analysis assessed the blue side. On the first step, condition was added to the model and was not significant $\left(R_{\text {change }}^{2}<.001, F_{\text {change }}<1\right)$, as was predicted. On the second step, participant codes accounted for $88.4 \%$ of the variance $\left[F_{\text {change }}(48,221)=35.3, p<.0001\right]$ : The participants showed substantial variation in their trajectories. On the third step, the main effect of rStep accounted for an additional $1.4 \%$ of the total variance and $12.4 \%$ of the subject variance $\left[B=3.3, F_{\text {change }}(1,49)=6.98, p=.011\right]$ : As rStep increased (toward the boundary), there was more deviation. Finally, on the fourth step, the interaction of rStep and condition accounted for no additional variance $\left(F_{\text {change }}<1\right)$.
We next asked whether the effect of rStep was driven by the stimuli adjacent to the boundary by excluding any data point with an rStep greater than -1 . It was possible that within-category differences would be observed only near the boundary, and we wanted to rule this out. Condition again was not significant $\left(R_{\text {change }}^{2}<.001, F_{\text {change }}<1\right)$, and participant accounted for $92.7 \%$ of the variance $\left[F_{\text {change }}(48,171)=45.45, p<.0001\right]$. Importantly, rStep was still significant, accounting for $0.7 \%$ of the total variance and $10.1 \%$ of the participant variance $[B=2.8$, $\left.F_{\text {change }}(1,49)=5.53, p=.023\right]$, and as before, the interaction accounted for none of the variance $\left(F_{\text {change }}<1\right)$.

On the green side, the results were similar. The first model examined all of the green rSteps. Condition accounted for no significant variance $\left(F_{\text {change }}<1\right)$. Participant codes accounted for an additional $88.1 \%$ of the variance $\left[F_{\text {change }}(48,229)=35.2, p<.0001\right]$. As before, the main effect of rStep significantly accounted for $2.3 \%$ of the total variance and $19.0 \%$ of the participant variance $\left[B=-3.9, F_{\text {change }}(1,49)=11.5, p=.0014\right]$. Here, as rStep increased (away from the boundary), the amount of deviation decreased. As before, the interaction was not significant, accounting for only $0.5 \%$ of the total variance and $4.4 \%$ of the participant variance $[B=3.7$, $\left.F_{\text {change }}(1,49)=2.26, p=.14\right]$.

We again asked whether the effect of rStep was driven by the stimuli adjacent to the boundary by excluding any data point with an rStep less than 1 . Condition accounted for none of the variance $\left(F_{\text {change }}<1\right)$, and participants accounted for $93.7 \%\left[F_{\text {change }}(48,179)=56.6, p<.0001\right]$. rStep accounted for a small but significant $0.5 \%$ of the total variance and $8.7 \%$ of the participant variance $[B=$ $\left.-2.25, F_{\text {change }}(1,49)=4.66, p=.036\right]$. The interaction 
was again not significant, accounting for only $0.1 \%$ of the total variance and $2.1 \%$ of the participant variance $\left[F_{\text {change }}(1,49)=1.0, p=.32\right]$.

Finally, a test of bimodality examined the distribution of deviations at each integer rStep. A unimodal distribution was observed at each rStep (Kolmogorov-Smirnov test: $p<.05$; further details are available from the first author).

\section{DISCUSSION}

This experiment demonstrated that color categorization is sensitive to continuous differences in hue within the category: As the hue approached the boundary, mouse trajectories increasingly deviated toward the competitor color. This was true despite a conservative analysis that considered only trials on which the correct color was selected, eliminated between-subjects variability in boundary, and ignored steps adjacent to the boundary. Thus, fine-grained differences in hue are available simultaneously with categories, in a categorization task.

Such effects could arise in at least two ways. First, continuous hue could be maintained throughout processing, in terms of raw sensory values. Second, fine-grained details in hue could be available, in terms of differences in category goodness (e.g., that hue is +2 steps from prototypical blue). Although these two alternatives cannot be distinguished at present, it remains that some version of continuous hue is present in a task emphasizing categorical responding.

Methodologically, the present work demonstrates that mouse movements are rich enough to index gradient representations as a function of gradations in the input, even when the overt response is constant. More specific to issues raised in color perception, these methods also offer new avenues in which to examine the role of language in color perception, allowing us to test whether factors such as visual field (hemispheric asymmetries) or the set of labels offered by the language affect the magnitude and outcome of these competitive processes.

This work also emphasizes that beyond the parallel maintenance of two kinds of information, there is parallelism within the categorical level. Our data suggest that two color categories were considered in parallel (to various degrees) and reflect a probabilistic activation of multiple possible responses.

These results parallel the results from speech perception (McMurray et al., 2008; McMurray, Tanenhaus, \& Aslin, 2002), and suggest that parallel activation and gradiency, not discrete categories, are the norm across perceptual categorization. Such processes can be modeled by timevarying dynamical systems (e.g., McClelland \& Elman, 1986). However, whereas speech is fundamentally dynamic, color is not considered to be time-varying. Yet the present results suggest that dynamic processes may best characterize the perception of static stimuli as well. This kind of system may be advantageous in real environments where there are constantly changing variables such as illumination, surface reflection, and visual angle. Such a mechanism would allow continuous hue to be used for things like color constancy (McCann, McKee, \& Taylor, 1976), while being sensitive to linguistic (Rosch, 1975; Siok et al., 2009) and other (Goldstone, 1995; Hansen, Olkkonen, Walter, \& Gegenfurtner, 2006) categories as the system balances between competing demands. At the same time, it would allow for categories to emerge over time (Fonteneau \& Davidoff, 2007) and even affect other processes (Daoutis, Pilling, \& Davies, 2006; Hampton et al., 2005; Kulikowski \& Vaitkevicius, 1997). Thus, a dynamic competitive approach may offer a better way to characterize the complex relationship between hue and category than do models emphasizing discrete categories and categorical perception.

\section{AUTHOR NOTE}

This project was supported by NIH Grant DC008089 to B.M. The authors thank Dan McEchron for assistance with data collection, Michael Spivey and Cathleen Moore for comments on previous drafts, and Jake Oleson for his statistical help. We also thank two anonymous reviewers for their helpful comments. Correspondence concerning this article should be addressed to S. Huette, School of Social Sciences, Humanities, and Arts, University of California, 5200 North Lake Road, Merced, CA 95343 (e-mail: shuette@ucmerced.edu).

\section{REFERENCES}

Bornstein, M. H., \& Korda, N. O. (1984). Discrimination and matching within and between hues measured by reaction times: Some implications for categorical perception and levels of information processing. Psychological Research, 46, 207-222.

CoHen, J., \& CoHEN, P. (1983). Applied multiple regression/correlation analysis for the behavioral sciences (2nd ed.). Hillsdale, NJ: Erlbaum.

Cohen, J., MacWhinney, B., Flatt, M., \& Provost, J. (1993). PsyScope: An interactive graphic system for designing and controlling experiments in the psychology laboratory using Macintosh computers. Behavior Research Methods, Instruments, \& Computers, 25, 257-271.

Dale, R., Kehoe, C., \& Spivey, M. J. (2007). Graded motor responses in the time course of categorizing atypical exemplars. Memory $\&$ Cognition, 35, 15-28.

Daoutis, C. A., Pilling, M., \& Davies, I. R. L. (2006). Categorical effects in visual search for colour. Visual Cognition, 14, 217-240.

Drivonikou, G. V., Kay, P., Regier, T., Ivry, R. B., Gilbert, A. L., Franklin, A., \& Davies, I. R. L. (2007). Further evidence that Whorfian effects are stronger in the right visual field than the left Proceedings of the National Academy of Sciences, 104, 1097-1102.

Farmer, T. A., Anderson, S. E., \& Spivey, M. J. (2007). Gradiency and visual context in syntactic garden-paths. Journal of Memory \& Language, 57, 570-595.

FontenEAU, E., \& DAVIDOFF, J. (2007). Neural correlates of colour categories. NeuroReport, 18, 1323-1327.

Franklin, A., Drivonikou, G. V., Bevis, L., Davies, I. R. L., Kay, P., \& REgIER, T. (2008). Categorical perception of color is lateralized to the right hemisphere in infants, but to the left hemisphere in adults. Proceedings of the National Academy of Sciences, 105, 3221-3225.

Freeman, J. B., Ambady, N., Rule, N. O., \& Johnson, K. L. (2008). Will a category cue attract you? Motor output reveals dynamic competition across person construal. Journal of Experimental Psychology: General, 137, 673-690.

Gilbert, A. L., Regier, T., Kay, P., \& Ivry, R. B. (2006). Whorf hypothesis is supported in the right visual field but not the left. Proceedings of the National Academy of Sciences, 103, 489-494.

Goldstone, R. L. (1995). The effects of categorization on color perception. Psychological Science, 6, 298-304.

Hampton, J., Estes, Z., \& Simmons, C. L. (2005). Comparison and contrast in perceptual categorization. Journal of Experimental Psychology: Learning, Memory, \& Cognition, 31, 1450-1476.

Hansen, T., Olkkonen, M., Walter, S., \& Gegenfurtner, K. R. (2006). Memory modulates color appearance. Nature Neuroscience, 9, 1367-1368. 
Kulikowski, J. J., \& Vaitkevicius, H. (1997). Colour constancy as a function of hue. Acta Psychologica, 97, 25-35.

Liberman, A. M., Harris, K. S., Hoffman, H. S., \& Griffith, B. C. (1957). The discrimination of speech sounds within and across phoneme boundaries. Journal of Experimental Psychology, 54, 358368.

McCann, J. J., McKee, S. P., \& TAYlor, T. H. (1976). Quantitative studies in retinex theory: A comparison between theoretical predictions and observer responses to the "color Mondrian" experiments. Vision Research, 16, 445-458.

McClelland, J. L., \& Elman, J. L. (1986). The TRACE model of speech perception. Cognitive Psychology, 18, 1-86.

McMurray, B., Aslin, R. N., Tanenhaus, M. K., Spivey, M. J., \& SuBIK, D. (2008). Gradient sensitivity to within-category variation in speech. Journal of Experimental Psychology: Human Perception \& Performance, 34, 1609-1631.

McMurray, B., Tanenhaus, M. K., \& Aslin, R. N. (2002). Gradient effects of within-category phonetic variation on lexical access. Cognition, 86, B33-B42.

McMurray, B., Tanenhaus, M. K., \& Aslin, R. N. (2009). Withincategory VOT affects recovery from "lexical" garden paths: Evidence against phoneme-level inhibition. Journal of Memory \& Language, 60, 65-91.

Oden, G. C., \& MASSARo, D. W. (1978). Integration of featural information in speech perception. Psychological Review, 85, 172-191.

Pilling, M., Wiggett, A., Özgen, E., \& Davies, I. R. L. (2003). Is color "categorical perception" really perceptual? Memory \& Cognition, 31, 538-551

Pisoni, D. B., \& TAsh, J. (1974). Reaction times to comparisons within and across phonetic categories. Perception \& Psychophysics, 15, 285 290.

Roberson, D., \& DAVIDOFF, J. (2000). The categorical perception of colors and facial expressions: The effect of verbal interference. Memory \& Cognition, 28, 977-986.

Roberson, D., Hanley, J. R., \& PAK, H. (2009). Thresholds for color discrimination in English and Korean speakers. Cognition, 112, 482487.

Rosch, E. (1975). The nature of mental codes for color categories. Journal of Experimental Psychology: Human Perception \& Performance, 1, 303-322.

SchmidT, T. (2002). The finger in flight: Real-time motor control by visually masked color stimuli. Psychological Science, 13, 112-118.

Schouten, B., Gerrits, E., \& VAn Hessen, A. (2003). The end of categorical perception as we know it. Speech Communication, 41, 71-80.

Siok, W. T., Kay, P., Wang, W. S. Y., Chan, A. H. D., Chen, L., Luke, K.-K., \& TAN, L. H. (2009). Language regions of brain are operative in color perception. Proceedings of the National Academy of Sciences, 106, 8140-8145.

Spivey, M. J., Grosjean, M., \& Knoblich, G. (2005). Continuous attraction toward phonological competitors. Proceedings of the National Academy of Sciences, 102, 10393-10398.

UchiKaWA, K., \& SHINODA, H. (1996). Influence of basic color categories on color memory discrimination. Color Research \& Application, 21, 430-439.

(Manuscript received April 19, 2009; revision accepted for publication January 7, 2010.) 